\title{
Research on the Teaching of Probability Theory and Mathematical Statistics for Non-statistics Majors
}

\author{
Feng $\mathrm{WANG}^{1,{ }^{*}}$ and Xiao-ping $\mathrm{XU}^{2}$ \\ ${ }^{1}$ School of Mathematics and Statistics, Xi'an Jiaotong University, Xi'an 710049, China \\ ${ }^{2}$ School of sciences, Xi'an University of Technology, Xi'an 710054, China \\ ${ }^{*}$ Corresponding author
}

Keywords: Probability theory and mathematical statistics, Teaching, Method, Effect.

\begin{abstract}
Probability theory and mathematical statistics is a complicated course, which has the characteristics of systematic and logical. Currently, the teaching of probability theory and mathematical statistics for Non-statistics Majors is an important problem in engineering mathematics education of our country. This paper puts forward the idea of introducing mathematical experiment method into the teaching process, advocating the use of the concept map, using computer assisted instruction method, instilling the idea of mathematical modeling, introducing the idea of statistical modeling and adding the topic teaching concept to optimize and enrich the teaching means and method, so as to stimulate the interest of students' learning probability theory and mathematical statistics to tap the potential of the students as far as possible. Accordingly, the teaching of the course is more effective, and the teaching quality is also improved.
\end{abstract}

\section{Introduction}

In today's society based on information and technology, people are faced with more opportunities and choices. People often need to make rational decisions based on a large amount of data in uncertain scenarios (random phenomena). Probability theory and mathematical statistics is to help people look at the world from a random point of view, and help people how to make a more correct and reasonable decision in the face of a variety of accidental or a large number of irregular data [1]. From 1933, Soviet mathematician Kolmogorov's landmark work "the basic concept of probability theory" to the present, probability theory and mathematical statistics and other disciplines is mutually penetrated, so it has a new development [2, 3].

The teaching of probability theory and mathematical statistics for non-statistics majors has been an important part of higher mathematics education, and how to improve the teaching level has aroused wide concern in the educational circles [4]. Probability theory and mathematical statistics is not only necessary foundation of the study of follow-up courses and the theoretical research and practical work, but also plays important role in cultivating students' comprehensive ability and improving students' mathematical accomplishment [5]. As the study object of probability theory and mathematical statistics is special, compared with other mathematics curriculum, it has its unique concepts and methods. Students are often difficult to carry out the thinking and solving method in the learning process, a lot of students are unable to digest the knowledge, so it is very important to improve the teaching quality of probability theory and mathematical statistics.

\section{Introducing the Idea Method of Mathematical Experiment}

Mathematical experiment is a course which starting from the problem, through students' thinking and analysis, using mathematical software to solve the problem. The course is characterized by the combination of hand and brain, the idea can be verified by computer, the calculated results are directly showed by the data or the image. Problem theory and mathematical statistics is a strong practical course, some conclusions of the course can be verified by the computer, some conclusions can be solved by the computer simulation. Thereby, how to effectively combine them has become a 
key problem. Recommendations are as follows. (1) The interface of Matlab software is friendly, close to the windows standard interface, human-computer interaction is strong, the program dose not need to be compiled to run. It also has a strong computing power and excellent data visualization capabilities. In particular, it is suitable for the students who first contact with the software, which is beneficial to the teaching of the teachers. Thereby, Matlab software should be adopted by teachers in the teaching process. (2) The knowledge points should be combined. For example, graphics can be used to express the distribution law and the probability density, the simulation of Bernoulli experiment, Poisson distribution to approximate Binomial distribution, the verification of the law of large number and central limit theorems, the probability density function and parameter relation of three common distribution in statistics, the verification and simulation of the parameter point estimation and interval estimation, the simulation of general stochastic problems. A computer simulation method for generating other distributed variables by uniform distribution is advised to learn top students as homework, teachers can also provide or specify programming language reference for students to self-study. (3) Some content fit for programming, the final results are presented to the students. Some content fit for the scene programming in the classroom, and the effect is better. Some simulation is suitable for students to spent their time after school. In the field programming, the following point should be pay attention. Some function should be modularize, it is given in the form of function. It must try to write out the procedure looks like one thing to do is to explain.

\section{Rational Use of Concept Map}

In foreign countries, concept map as an effective strategy has been widely studied and applied. In China, there is less research on the concept map, especially in the application of mathematics teaching. Concept map is from the translation of the English "concept map", it is also called as the concept mapping. Generally, concept map includes four basic element, that is, nodes, connection, hierarchy and proposition. A node represents a concept. The connection indicates that there is a relationship between the two concepts. The hierarchy is the presentation of the concept. The proposition is the meaning relationship formed by the concepts. Concept map is a kind of scientific proposition, which shows the relationship the concepts, thus it is a space network structure connecting the basic concept. Probability theory and mathematical statistics has many basic concept, it is an intricate mathematical course. The structure of concept map itself determines that it is more important to emphasize the concept and principle, so it is suitable for probability theory and mathematical statistics. Therefore, in teachers' preparing lessons, if they use the concept map to analyze the curriculum system, sum up the teaching design ideas of their own curriculum system, analyze the key knowledge of the course unit and display some complex ideas, so it can provide more effectively the teaching contents. Concept map can expressed related concepts and principles in concise, it is a kind of concentrate for the knowledge. Using the concept map can make students understand the knowledge more deeply, the relationship between concepts more clearly, grasp the knowledge in the full view for the knowledge, that is to master the knowledge not only have more depth, width and breadth. So it can help students to construct knowledge actively to form good cognitive structure. In terms of acquiring knowledge, students is no longer direct possession of the textbook on the conclusion, or directly remember the conclusions analyzed by teachers, they make the knowledge become concept map to easy to store, understand, extract and use knowledge. Concept map is a kind of hierarchy network structure diagram. It organizes information in a logical way, so it can cultivate the ability of students' mathematical logic thinking. Concept map will be implicit knowledge explicit, which will help students to criticize themselves and other people's cognitive structure, so it is helpful to cultivate students' critical thinking. Concept map can also cultivate students' creative thinking, which is determined by the form of concept map itself. 


\section{Using Computer Assisted Teaching}

In the face of the general trend of the development of computer assisted instruction, if teachers introduce the computer knowledge to the teaching process for probability theory and mathematical statistics, it can fully utilize the characteristics of computing speed and high accuracy, students can use the computer language into some very simple programs storage on the disk for all kinds of integral operation, the sum of series and operation formula. In solving the specific problem, students only need to analyze the problem the requirements and problem-solving methods, and then call the appropriate procedure, the results can be obtained after the data are input. It can fully mobilize students' interest in learning, at the same time, it can also solve the problem of the limitation brought by the textbook, which can optimize the effect of teaching to enable students to better understand and grasp the basic theory and methods. If teachers can actively guide the students and introduce the computer to the teaching process for probability theory and mathematical statistics step by step, which can effectively improve learning effect for probability theory and mathematical statistics and the ability of using it to solve practical problems, which can strengthen students' computer knowledge and improve students' enthusiasm for learning computer. Of course, this also puts forward higher requirements for every mathematical teachers. Teachers should not only have a solid theoretical knowledge of mathematics, but also must try to learn some computer knowledge, and continuously improve the ability to use the computer to organize knowledge, let the computer continue to be deep the teaching of mathematical theory to actively prepare computer assisted teaching in college mathematics.

\section{Establishing the Consciousness of Mathematical Modeling}

In recent years, mathematical modeling teaching and competition has been developed in the domestic and international, it has greatly promoted the reform of mathematics teaching. Mathematical model is to get some objective reality of mathematical structure for abstraction and simplification of the prototype, it is to simplify the prototype model of reality by using mathematical symbols, mathematical formulas and numerical relationship, and the process of constructing a mathematical model for the practical problems is called as mathematical modeling. From the point of view of mathematics, mathematical modeling is a simulation of the search problem, give the irrelevant and retain their mathematical relationship to form a mathematical structure. In a broader sense, modeling is a technique, a method, a concept. Mathematical modeling has been widely used in science and engineering technology. As everyone knows, probability theory and mathematical statistics is very strong discipline, such as the ideas of probability, independent, relation, mathematical expectation, the law of large number, random thoughts, sampling, sample estimation, data compression idea, the principle of maximum likelihood, small probability principle, accept or deny the hypothesis at the significant level, regression, etc. It is important to teach students to think about the problems in the teaching process, such as the statistical method is good or bad, there is no right or wrong, which is very different from the traditional mathematics. In additional, probability theory and mathematical statistics contains a rich mathematical methods, such as modeling method, construction method, transformation method, quantitative method, analogy method, etc. In particular, modeling methods runs through the course of probability theory and mathematical statistics, such as classical probability, geometric probability, Bernoulli model, normal distribution, binomial distribution, various test of normal population, variance analysis, regression analysis, etc. It is also the starting point of our view that we can use mathematical modeling theory to guide the teaching of probability theory and mathematical statistics. Refining these methods in teaching is important to deepen the knowledge of students, form the skills of students' solving the problem, develop intelligence and improve the diathesis. Mathematical modeling is used to guide teaching, which is to strengthen the application of awareness, teaching should be oriented to the practical application of the computer and adopt modern teaching methods. 


\section{Integrating the Idea of Statistical Modeling}

In today's twenty-first century, whether it is the management of the national economy and the company, business decision, or scientific research is more and more dependent on the statistical analysis of the data. When massive data and complex information are faced, how to quickly and effectively find the law of the development and change of things is an important research topic. Statistical analysis software (such as SPSS, SAS, R language, etc) is as a tool in statistical modeling. In statistical modeling, using various statistical analysis methods analyzes batch data, then the model is established according to economic theory to fully reveal the factors behind the data to interpret economic phenomena. It can be said that statistical modeling perfectly combines statistical ideas, statistical methods, economic management theory and computer technology, and it can not only drive the analysis of statistical thinking oriented data, but also provide ideas and countermeasures for social economic management. Therefore, statistical modeling is introduced into the teaching process of probability theory and mathematical statistics to promote the course's teaching reform. Accordingly, it is important to improve students' statistical literacy and use it to solve practical problems. In modern society, the analysis, processing and application of the data can not be separated from the computer. Without mastering a statistical software, the analysis and processing of the data is out of the question. Therefore, teachers should pay great attention to students' proficiency in application of statistical software in the teaching process.

\section{Adding the Teaching Idea of Topic Type}

The phenomena of nature can be roughly divided into two categories, one is the determine phenomenon, the other is a random phenomenon. Probability is linked to the search for authenticity. In reality, it is full of uncertainty. Subject based integrated teaching is as a teaching task in a separate case. Each subject is focused on the instance, and it should be the life and production from our side. Students are interested in the teaching topic to recognize what is learning to be useful for solving practical problems, so their passion are natural to rise up for the learning. Interest is the best teacher, it can arouse students' interest to study, so learning effect is certainly good to some extent. Examples of the topic are from the production and life. In order to deal with these problems, teachers should explain clearly to the students to guide them to put forward some specific ways to solve them. Accordingly, through learning these practical examples, students can gradually improve the ability to solve practical problems. In the implementation of the teaching, it should pay attention to decompose the difficult problems and do a work in guiding. Otherwise, the teaching effect will be greatly reduced. In general, students are interested in the subject, but they also feel that there is a certain difficulty. Therefore, teachers should analyze the difficulties in advance to guide students to complete the system design. That is, teachers should guide students to think about how to convert practical problems into mathematical problems. After such a guide is done to decompose the difficulty, the design idea of the subject has been very clear in the students' mind, so the system design will be smoothly done or easy solved. As long as the difficulty is perfectly decomposed, students will soon be solved using corresponding knowledge.

\section{Summary}

In the teaching for the course of probability theory and mathematical statistics, teachers should change the past teaching methods, boldly explore the teaching reform methods to enhance the quality of teaching to enable students to understand the basic idea of probability theory and mathematical statistics, grasp the probability statistical inference skills and improve their ability to analyze and solve problems. 


\section{Acknowledgment}

This research was financially supported by the Teaching Research Project of Xi'an University of Technology under Grant No. xjy1766.

\section{References}

[1] B.P. Tian, Y. Wang, Exploration and thoughts for teaching of probability and statistics in the institute of technology, Colg. Math. 21(2005): 17-21.

[2] X.H. Chen, L.J. Chen, S.H. Gong, Concept map: an effective tool for the cultivation of creative thining, CHN Mod. Educ. Equip. 4(2007): 137-139.

[3] Z.X. Ni, M. Chen, Modern teaching method of probability and statistics of technology focusing on statistics idea, Colg. Math. 20(2004): 21-23.

[4] F.P. Feng, J.X. Cui, Exploration and improvement of the teaching of probability theory and mathematical statistics, J. Sci. Teachers’ Coll. Univ. 24(2004) : 82- 83.

[5] D.T. Li, Integrating mathematical modeling into the main courses of mathematics, J. Eng. Math. 22(2005): 2-7. 